\title{
Efecto del tipo de cambio sobre el déficit fiscal: un modelo estocástico de reversión a la media con saltos para el caso colombiano
}

\author{
Recibido: septiembre, 09 de 2017 - Aprobado: junio, 19 de 2018
}

DoI: http://dx.doi.org/10.12804/revistas.urosario.edu.co/economia/a.7194

\author{
Clark Granger-Castaño*
}

Jhon Fredy Moreno-Trujillo ${ }^{\dagger}$

Francisco Venegas-Martínez $\ddagger$

\section{Resumen}

Los métodos más utilizados para evaluar el impacto del tipo de cambio sobre el déficit fiscal son deterministas y se basan en las elasticidades de cada una de las variables que inciden en el déficit. Esto permite tener una idea muy limitada de la magnitud y dirección de futuros choques. Esta investigación desarrolla un modelo estocástico para evaluar el impacto del tipo de cambio sobre el déficit fiscal en un ambiente de incertidumbre. Para ello, la dinámica de la tasa de depreciación del tipo de cambio se modela como un proceso con reversión a la media combinado con saltos de Poisson. A partir del modelo teórico propuesto, se realizan simulaciones de Montecarlo de las proyecciones del déficit del gobierno nacional central de Colombia (GNCC). Esta simulación proporciona estimaciones de las metas fiscales considerando los efectos aleatorios del tipo de cambio. Por último, a partir de las proyecciones obtenidas, se estima una senda de la deuda del gobierno con

* Banco de la República - Colombia. Correo electrónico: cgrangca@banrep.gov.co

+ Universidad Externado de Colombia. Correo electrónico: jhon.moreno@uexternado.edu.co

‡ Instituto Politécnico Nacional (México). Correo electrónico: fvenegas1111@yahoo. com.mx

Cómo citar este artículo: Granger-Castaño, C., Moreno-Trujillo, J. F., \& Venegas-Martínez, F. (2018). Efecto del tipo de cambio sobre el déficit fiscal: un modelo estocástico de reversión a la media con saltos para el caso colombiano. Revista de Economía del Rosario, 21(2), 193-218. DoI: http:/ /dx.doi.org/10.12804/revistas.urosario.edu.co/economia/a.7194 
base en la tasa de depreciación del tipo de cambio, la cual es útil para la planeación del gasto del GNCC y para el planteamiento de las metas fiscales.

Palabras clave: déficit fiscal, tipo de cambio, procesos estocásticos, simulación de Montecarlo. Clasificación JEL: H62, O24, C63.

\title{
Effects of the Exchange Rate on the Fiscal Deficit: A Stochastic Mean-Reverting Jump-Diffusion Model for the Colombian Case
}

\begin{abstract}
The most used methods to assess the impact of the exchange rate on the fiscal deficit are deterministic and are based on the elasticities of each of the variables affecting the deficit. This provides a very limited idea of the magnitude and direction of future shocks. This research develops a stochastic model useful to evaluate the impact of the exchange rate on the fiscal deficit in an environment of uncertainty. To do this, the dynamics of the exchange rate depreciation is driven by a mean-reverting jump-diffusion process. By using the theoretical proposed model, Monte Carlo simulations of the projections of the deficit of the Central National Government of Colombia (CNGC) are carried out. The simulation provides estimates of fiscal targets considering the random effects of the exchange rate. Finally, from the obtained projections, a path of government debt is estimated based on the depreciation of the exchange rate, which is useful for the planning of the CNGC expenditure and for the statement of fiscal goals.
\end{abstract}

Keywords: Fiscal deficit, exchange rate, stochastic processes, Monte Carlo simulation. JEL Classification: H62, O24, C63

\section{Efeito do tipo de câmbio sobre o déficit fiscal: um modelo estocástico de reversão à média com saltos para o caso Colombiano}

\section{Resumo}

Os métodos mais utilizados para avaliar o impacto do tipo de câmbio sobre o déficit fiscal são deterministas e se baseiam nas elasticidades de cada uma das variáveis que incidem no déficit. Isto permite ter uma ideia muito limitada da magnitude e direção dos futuros choques. Esta pesquisa desenvolve um modelo estocástico para avaliar o impacto do tipo de câmbio sobre o déficit fiscal em um ambiente de incerteza. Para isso, a dinâmica da taxa de depreciação do tipo de câmbio se modela como um processo com reversão à média combinado com saltos de Poisson. A partir do modelo teórico proposto se realizam simulações Monte Carlo das projeções do déficit do Governo Nacional Central da Colômbia (GNCC). Esta simulação proporciona estimações das metas fiscais considerando os efeitos aleatórios do tipo de câmbio. Por último, a partir das projeções obtidas estima-se uma senda da dívida do Governo com base na taxa de depreciação do tipo de câmbio, a qual é útil para o planejamento do gasto do GNCC e para o planejamento das metas fiscais.

Palavras-chave: déficit fiscal, tipo de câmbio, processos estocásticos, simulação Monte Carlo. Classificação JEL: H62, O24, C63. 


\section{Introducción}

El déficit fiscal y la deuda pública son variables utilizadas para medir el desempeño y dirección de la política fiscal en un período determinado. En este sentido, el estudio de los efectos del tipo de cambio sobre el déficit fiscal y la deuda pública es un asunto de importancia para el diseño de las políticas fiscal y de gasto. Al respecto, Penati (1983) examina la relación entre el tipo de cambio y la política fiscal. El autor concluye, mediante ejercicios de equilibrio neoclásicos, que una política fiscal expansiva genera aumentos en la producción y en los precios, los cuales se equilibrarán con una apreciación del tipo de cambio. Se destaca que esta política podría llevar a la producción más allá de su nivel potencial, por lo que la situación es sostenible solo en el corto plazo, ya que las expectativas de una reducción futura en producción y precios generarán una expectativa de depreciación en el tipo de cambio. Así mismo, Penati (1983) identifica que la relación entre tipo de cambio y política fiscal depende de los movimientos del producto real y los precios, los cuales en el largo plazo están relacionados con la estructura productiva y los salarios, así como con la evolución del tipo de cambio.

Por otro lado, el trabajo de Rodríguez y Venegas-Martínez (2010) examina una relación entre el tipo de cambio y el déficit fiscal con el propósito de identificar los efectos de la tasa de depreciación del tipo de cambio sobre el balance fiscal del gobierno mexicano. Los autores realizan ejercicios de simulación de Montecarlo de las fluctuaciones del tipo de cambio peso mexicano-dólar americano y generan una trayectoria del balance fiscal. Los autores encuentran que el modelo explica de manera adecuada los datos observados en el pasado en comparación con diversos modelos deterministas. Para su comprobación dividen los datos observados en dos ventanas de tiempo, una en la década de los noventa y otra en la primera década del siglo xx, hallando que el modelo propuesto genera resultados más consistentes en ambas ventanas.

Así mismo, Westphal y Rother (2011) analizan el efecto de la deuda pública sobre el crecimiento del producto per capita de doce países europeos durante los últimos 40 años. El ejercicio es realizado a partir de un modelo de datos panel. Los autores reportan evidencia de efectos no lineales y revelan una relación cóncava entre las variables con un punto de inflexión para niveles de deuda como porcentaje del PIв entre el $90 \%$ y $100 \%$, lo que significa que la deuda pública se asocia, en promedio, con tasas de crecimiento más bajas a largo plazo en los niveles de deuda por encima de ese rango. Además, destacan que esta relación es no lineal con la inversión pública, la cual está relacionada con el tipo de cambio para países productores de petróleo. Por su parte, el trabajo de Agnello et al. (2013) busca medir los efectos de la 
política fiscal discrecional evaluando el efecto de esta sobre el gasto privado a través de un enfoque econométrico que utiliza modelos de datos panel de países miembros de la oECD. Los autores encuentran que la política fiscal expansiva impulsa el crecimiento en el corto plazo, pero lo deteriora en el mediano plazo. También, señalan que los efectos varían dependiendo de las condiciones económicas de un país, las cuales son descritas por el grado de apertura comercial y el nivel del tipo de cambio. Por otro lado, la investigación de Bouakez et al. (2014) busca medir los efectos macroeconómicos de la política fiscal utilizando una metodología empírica alternativa que relaja las restricciones de identificación utilizadas comúnmente en la literatura SVAR. Estos autores encuentran que la política fiscal expansiva es más efectiva que la disminución de impuestos con fines de dinamizar el producto interno bruto.

Igualmente, en el trabajo de Bouakez y Eyquem (2015) se contrastan los resultados de la teoría tradicional de Mundell-Fleming con los modelos dinámicos de equilibrio general que sostienen que un aumento en el déficit fiscal genera una apreciación en el tipo de cambio real. Para ello proponen un modelo de una economía pequeña y abierta basado en: mercados financieros incompletos e imperfectos, rigideces de precios y una política monetaria no agresiva. El modelo arroja como resultado una depreciación leve del tipo de cambio real, en contravía de los modelos tradicionales.

Otros enfoques que se han utilizado con buenos resultados para el pronóstico del tipo de cambio se encuentran en Jalil y Misas (2007), Ni y Yin (2009), Nag y Mitra (2002), Kuan y Liu (1995) y Tenti (1996), entre muchos otros. En particular Jalil y Misas (2007) contrastan diferentes especificaciones lineales y no lineales, incluyendo a las redes neuronales artificiales (RNA), ajustadas a la variación porcentual diaria del tipo de cambio utilizando funciones de costo simétricas y funciones de pérdida asimétricas. Sus resultados muestran que las RNA permiten obtener mejores pronósticos con ambos tipos de funciones de costos. Los autores también destacan que cuando se evalúan los pronósticos con funciones asimétricas, el modelo no lineal ofrece mejores resultados.

Por otro lado, la aplicación de procesos de difusión combinados con saltos para modelar el comportamiento estocástico de los precios de activos financieros inicia con las investigaciones pioneras de Merton (1976), Ball y Torous (1983), Aase (1988) y Bates (1988). Así mismo, el modelado de la dinámica aleatoria de diversas variables, incluyendo al tipo de cambio, mediante procesos estocásticos de difusión con saltos, se puede encontrar en: Vallejo-Jiménez et al. (2017), Venegas-Martínez et al. (2013), Branger y Larsen (2013), Duffie (2001), Schönbucher (2003), Johannes (2004), Duffie (2005) y Venegas-Martínez (2001, 2006, 2009). 
Por otro lado, Akgiray y Booth (1988) resaltan la conveniencia de modelar el tipo de cambio a través de procesos estocásticos de difusión con saltos. Estos autores modelan la dinámica del marco alemán, la libra esterlina y el franco francés contra el dólar americano, encontrando que el enfoque propuesto es superior a la combinación de procesos normales discretos. En el trabajo de Núñez y Ortega (2011), varios modelos paramétricos con saltos son desarrollados para la descripción del tipo de cambio peso mexicanodólar americano. Los autores reportan que los modelos en tiempo continuo con saltos de Poisson permiten una mejor descripción del comportamiento de dicha variable. Igualmente, Jiang (1998) propone un enfoque de inferencia directa para la estimación de los parámetros de los procesos de difusión con saltos en tiempo continuo a partir de datos observados en forma discreta. Este enfoque sugiere que los saltos son componentes importantes de la dinámica, incluso en presencia de heterocedasticidad condicional y reversión a la media. Por último, Beinea y Laurent (2003) examinan varios tipos de cambio (contra el dólar estadounidense) que han estado sujetos a intervenciones directas del banco central. Para ello, se mezcla la distribución normal con saltos para modelar en la dinámica del tipo de cambio y esta distribución funciona adecuadamente para la estimación de la persistencia de shocks de volatilidad.

El presente trabajo desarrolla un modelo estocástico útil para evaluar el impacto del tipo de cambio sobre el déficit fiscal en un ambiente de riesgo cambiario. La dinámica de la tasa de depreciación del tipo de cambio se describe como un proceso con reversión a la media combinado con un proceso de saltos de Poisson. En una primera etapa se utiliza un modelo lineal, AR(1), para calibrar el componente de reversión a la media. Es importante destacar que, si se utiliza únicamente este tipo de modelos, en general, no es posible describir ni pronosticar adecuadamente la dinámica del tipo de cambio, como señalan Jalil y Misas (2007) y Kuan y Liu (1995). En una segunda etapa se agrega un componente de saltos de Poisson para ajustar las innovaciones (exceso de curtosis y heterocedasticidad). Claramente, la mezcla de especificaciones lineales con saltos se aleja del mundo normal y lineal. Este tipo de modelos ofrece la ventaja de proporcionar expresiones analíticas del proceso de interés (el tipo de cambio). La consideración de un modelo estocástico para la dinámica del tipo de cambio hace que el análisis posterior del déficit fiscal sea más general que el enfoque tradicional. En este sentido, el modelo estocástico propuesto proporciona una dinámica más realista para el tipo de cambio (cf. Núñez y Ortega, 2011; Rodríguez y Venegas-Martínez, 2010; Beinea y Laurent, 2003). 
A partir del modelo estocástico propuesto, se realizan simulaciones de Montecarlo de las proyecciones del déficit fiscal. La simulación genera estimaciones de las metas fiscales considerando los efectos aleatorios del tipo de cambio. Por último, a partir de las proyecciones obtenidas, se estima una senda de la deuda pública con base en la tasa de depreciación del tipo de cambio, la cual es útil para la planeación del gasto del GNCC y para el planteamiento de las metas fiscales que se proyectan de manera anual.

El presente trabajo está organizado como sigue: en la sección 1 se analiza el déficit fiscal en Colombia en el período 2000-2015; en la sección 2 se describe la dinámica del tipo de cambio en Colombia durante 2000-2015, destacando el mecanismo de la tasa de depreciación del tipo de cambio de mantenerse cerca de su promedio y la presencia de saltos bruscos e inesperados; en la sección 3 se presenta el modelo estocástico de déficit fiscal y la metodología de estimación de los parámetros de dicho modelo; en el transcurso de la sección 4 se realiza una simulación de Montecarlo de las metas fiscales considerando los efectos aleatorios del tipo de cambio; a través de la sección 5 se efectúan proyecciones de la senda de la deuda del gobierno con base en la tasa de depreciación del tipo de cambio; y, por último, se exponen las conclusiones y se destacan las limitaciones.

\section{Análisis del déficit fiscal en Colombia 2000-2015}

En la presente investigación para el cálculo del déficit fiscal se considera exclusivamente el GNCC. Esto por dos razones: primero, porque la deuda externa es afectada por la variación del tipo de cambio y es emitida en su mayoría por el gobierno central; y, segundo, por la importancia del balance del gobierno central en términos de la medición del desempeño de la política fiscal, ya que la meta de la regla fiscal se plantea sobre este resultado. Para la elaboración de estos cálculos, se utilizan las series mensuales de ingresos del GNCC, las cuales en su desagregación contienen los ingresos corrientes, ingresos de capital y fondos especiales. Los gastos del GNCC contienen los gastos de funcionamiento, gastos en intereses internos y externos, así como la inversión. La serie de la deuda bruta del GNCC se presenta en la figura 1 y está desagregada en deuda interna y externa.

Así mismo, en la figura 1 se ilustran los ingresos y gastos del GNCC para el período de estudio. Se observa una tendencia creciente de los ingresos, con excepción de 2010 cuando por motivo de la caída del precio internacional del petróleo se redujo el nivel de rentas (ingresos petroleros no tributarios) 


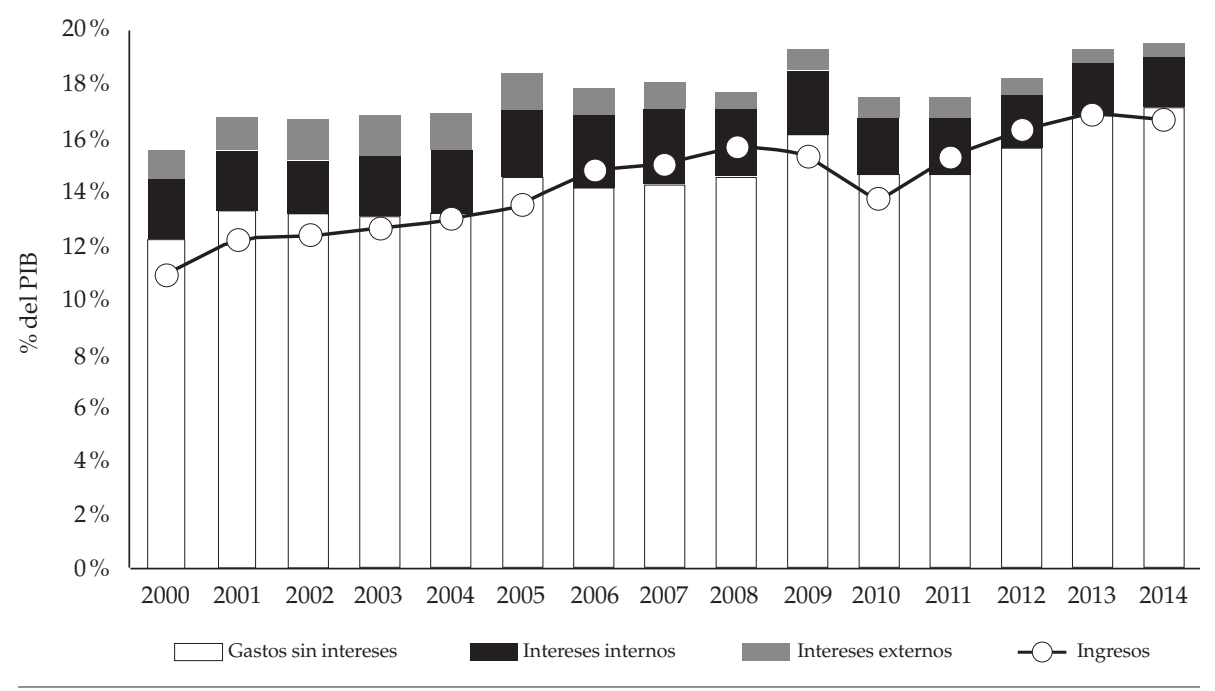

Figura 1. Ingresos y gastos del gobierno nacional central

Fuente: elaboración de los autores con datos del Ministerio de Hacienda y Crédito Público.

provenientes de ese sector de manera sustancial. En los gastos se ha mantenido una tendencia estable, y dentro de los componentes de este se identifican, como el de mayor crecimiento, los gastos sin intereses que comprenden funcionamiento e inversión, y desagregando aún más se encuentra el crecimiento destacado del rubro de pensiones que se incrementó del 1,8\% del PIB en 2000 al 3,6\% en 2014. A su vez, el rubro de inversión tuvo un crecimiento de más del doble al pasar del 1,3\% del pib en 2000 al 3\% en 2014. Por el lado de los intereses se observa que tanto los internos como los externos han venido perdiendo participación, lo cual se explica, en parte, por el mejoramiento de las condiciones de acceso de crédito del país, el cual en los últimos años ha tenido tasas de financiamiento históricamente bajas.

Con respecto a la deuda bruta del GNCC, que incluye deuda intrapública, se observa en la figura 2 que esta ha tenido un comportamiento irregular con respecto de los ingresos y gastos. Se observa un pico en 2003 que corresponde a los años posteriores a la crisis financiera de finales del siglo xx, en la que el gobierno suscribió un acuerdo de ajuste macroeconómico con el Fondo Monetario Internacional (FMI). Además, se destaca la pérdida de participación de la deuda externa en la primera mitad de la década anterior, lo cual está explicado por el desarrollo del mercado interno para la renta fija pública y por una política adoptada por el gobierno de turno para realizar una reacomodación de las participaciones a favor de la deuda local. 


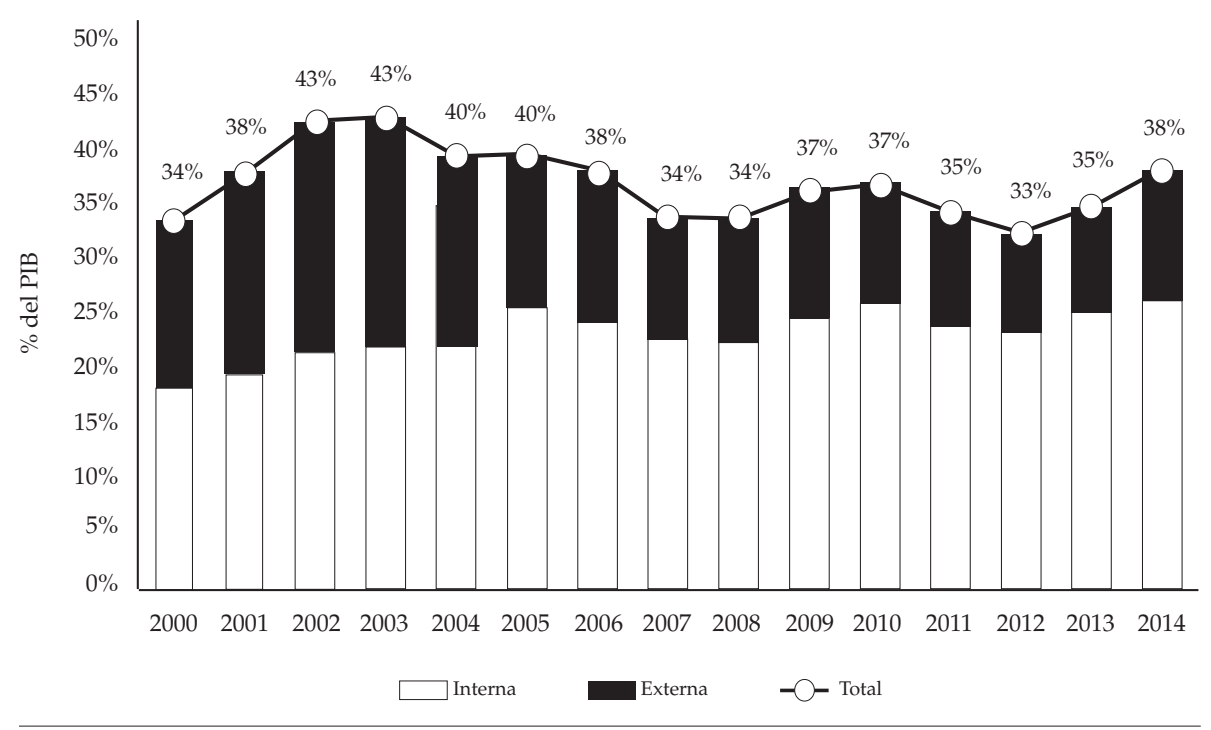

Figura 2. Deuda bruta del gobierno nacional central

Fuente: elaboración de los autores con datos del Banco de la República y del Ministerio de Hacienda.

\section{Dinámica del tipo de cambio en Colombia 2000-2015}

En 1992, en el contexto de la apertura comercial de Colombia, se optó por un régimen de bandas cambiarias en el que el banco central determinaba el rango donde podría oscilar el tipo de cambio y que, ante eventuales variaciones que excedieran los límites, la autoridad entraría a intervenir el mercado para corregirlo. A partir de octubre de 1999, cuando el país atravesaba por una crisis económica, se adoptó el régimen de libre flotación del tipo de cambio. En la figura 3 se representa la serie del tipo de cambio diario para el período de análisis considerado, que para este caso inicia en el año 2000, momento en el que arranca el régimen de flotación.

En la tabla 1 se muestra un resumen de las estadísticas descriptivas de la serie del tipo de cambio nominal y se observa que el valor máximo se encuentra cercano a los 3000 pesos colombianos, el cual se alcanzó en los años iniciales de la primera década del siglo xx. Esto obedece al período de la poscrisis hipotecaria, cuando Colombia tuvo dificultades cambiarias que también acarreaban varios países de la región, lo cual motivó al gobierno a solicitar una línea de crédito al FMI para cumplir con sus obligaciones de deuda externa. 


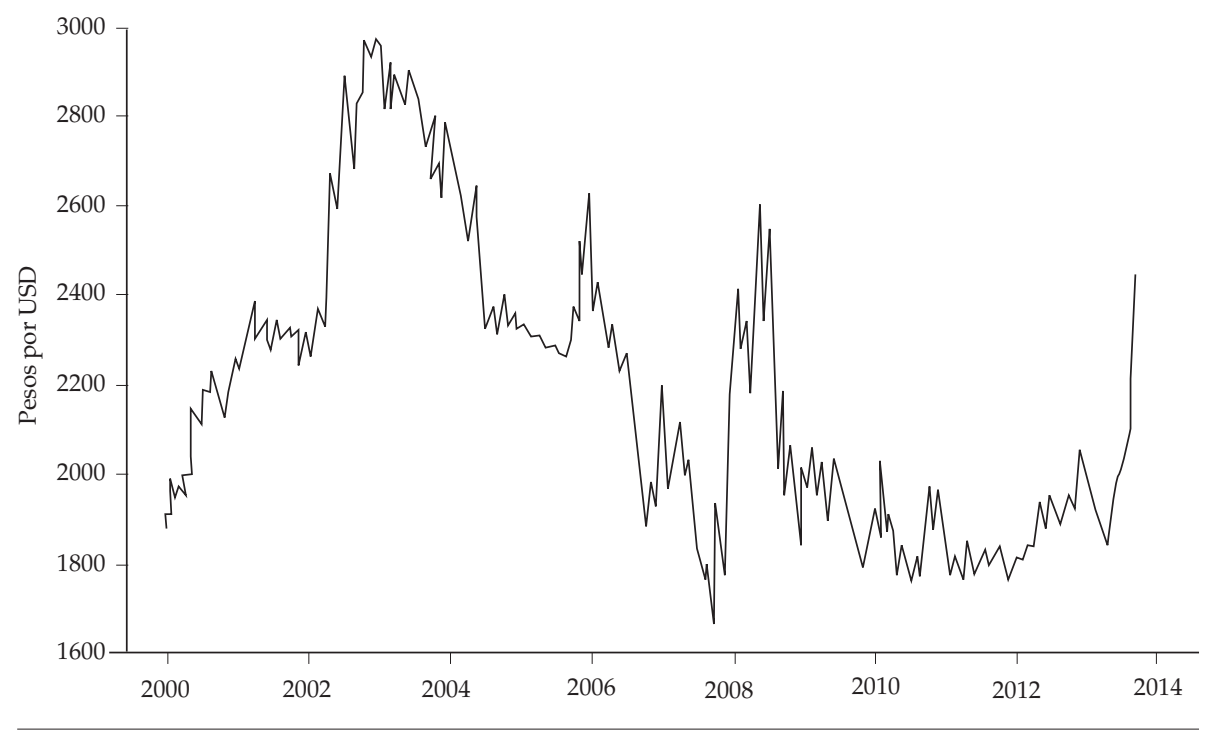

Figura 3. Tipo de cambio nominal pesos por dólares americanos (diario)

Fuente: elaboración de los autores con datos del Banco de la República.

Tabla 1. Estadísticas descriptivas del tipo de cambio nominal

\begin{tabular}{lr}
\hline Mediana & 2157,75 \\
Media & 2179,42 \\
Mínimo & 1652 \\
Máximo & 2969 \\
Desviación estándar & 327,03 \\
Varianza & 106962,20 \\
Sesgo & 0,66 \\
Curtosis & $-0,49$ \\
\hline
\end{tabular}

Fuente: elaboración de los autores con datos del Banco de la República.

Para el desarrollo del modelo y la metodología propuesta, se considera la serie de la tasa de depreciación del tipo de cambio, la cual es calculada a partir de los rendimientos logarítmicos de la serie. En la figura 4 se presenta la dinámica de la tasa de depreciación, la cual tiene una desviación estándar de 0,0051 durante el período observado. 
Así mismo, en la figura 4 se destaca un período de volatilidad entre 2008 y 2010, motivado por la crisis financiera internacional. En este período se encuentran la mayoría de las observaciones que exceden considerablemente tres desviaciones estándar (saltos).

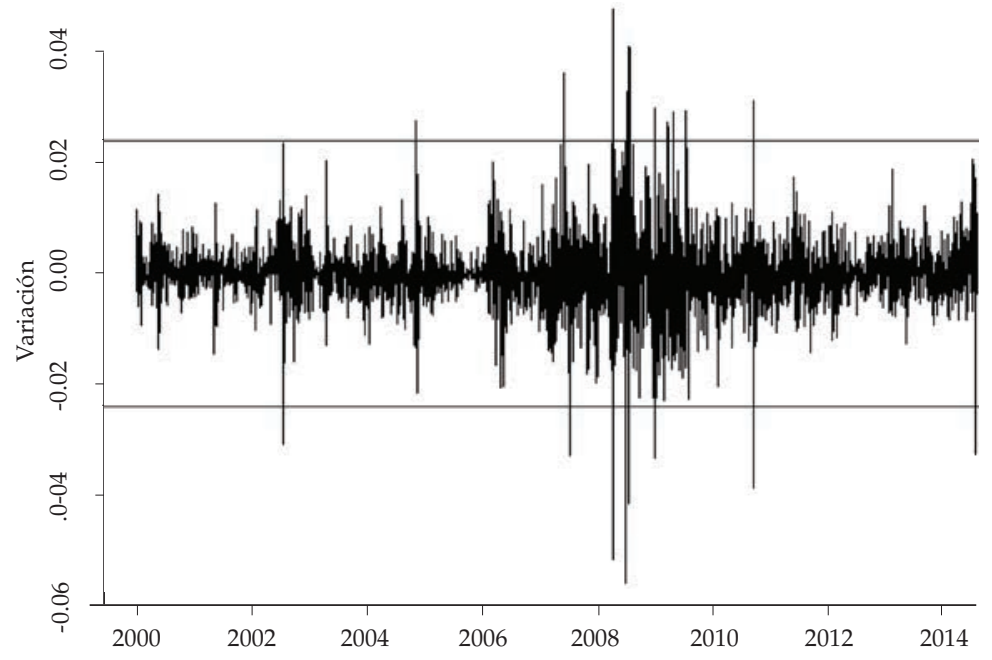

Figura 4. Tasa de depreciación diaria del tipo de cambio nominal

Las bandas consideradas están a tres desviaciones estándar de la media.

Fuente: elaboración de los autores con datos del Banco de la República.

Con respecto a las características de la tasa de depreciación, se realizaron algunas pruebas para examinar sus condiciones de estacionariedad. De forma inicial se decidió hacer la prueba de Dickey-Fuller de raíz unitaria con el fin de determinar la estacionariedad de la serie de la tasa de depreciación; el resultado fue de un valor $p$ menor a 0,01 , con lo cual se rechaza la hipótesis nula de no estacionariedad. Este resultado era esperado al tratarse de una serie de rendimientos. Luego, se procedió a efectuar las pruebas de normalidad de Anderson-Darling y de Kolmogorov-Smirnov, y en ambos se obtiene un valor $p$ menor a 2,2E-16, el cual rechaza la hipótesis nula de normalidad. Además, como se observa en la figura 5, el análisis Q-Q confirma estos resultados. Los resultados de las pruebas ofrecen validez y relevancia a la metodología que se propondrá para la modelación de la fluctuación de los datos alrededor de la media, pero con saltos extremos aleatorios. 


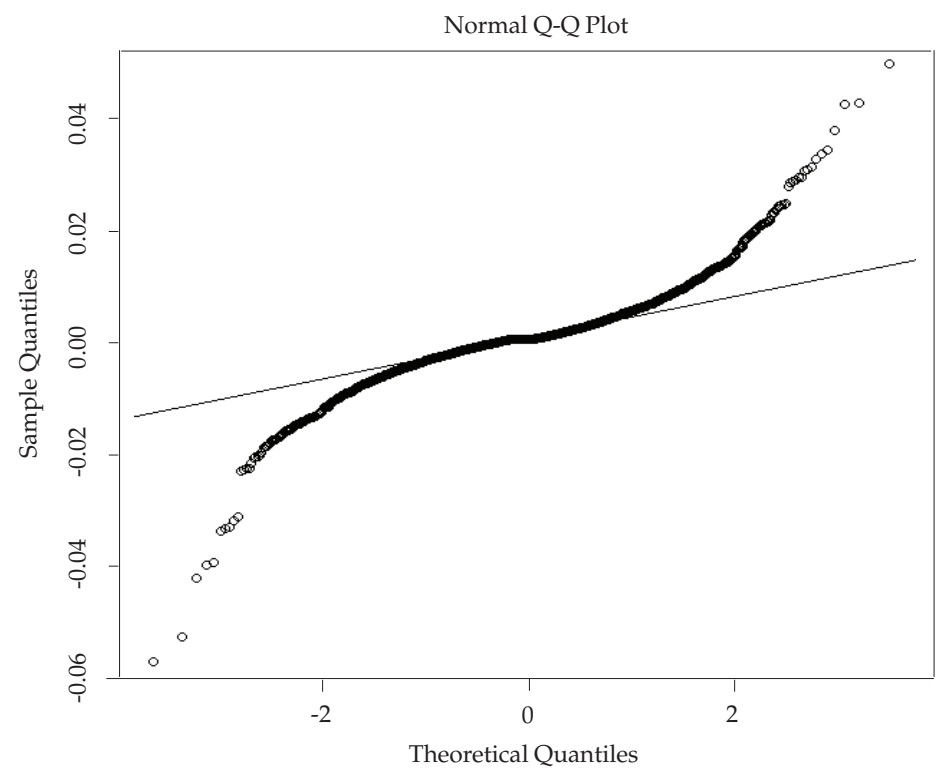

Figura 5. Gráfico $Q-Q$, variaciones del tipo de cambio

Fuente: elaboración de los autores con datos del Banco de la República.

\section{Modelo estocástico de déficit fiscal}

El déficit fiscal, $D_{t^{\prime}}$,resulta de la suma entre gastos sin intereses del gobierno, $G_{t^{\prime}}$ que incluye gastos de funcionamiento e inversión más el pago de intereses, $i_{t} B_{t-1}$, donde $i_{t}$ es la tasa de interés nominal de la deuda pública multiplicada por el saldo de la deuda en el período anterior, $B_{t-1}$, menos los ingresos totales del gobierno, $T_{t^{\prime}}$ como se muestra en la siguiente ecuación.

$$
D_{t}=G_{t}+i_{t} B_{t-1}-T_{t} .
$$

Esta forma de medición del déficit se denomina 'por encima de la línea'. Dicha expresión se puede reescribir como el déficit medido por la variación de la deuda pública, medición denominada 'por debajo de la línea', que mide el cambio en los activos y pasivos financieros, es decir, las fuentes de financiamiento del déficit gubernamental.

$$
D_{t}=B_{t}-B_{t-1}
$$


Bajo este esquema se tiene que una amortización se cuenta como un superávit y mayor deuda como un déficit. En la práctica, estas medidas arrojan resultados similares y las diferencias se dan por discrepancias en la inclusión de cuentas. ${ }^{1}$ Por lo tanto:

$$
B_{t}-B_{t-1}=G_{t}+i_{t} B_{t-1}-T_{t}
$$

En consecuencia, la deuda del gobierno en el período actual está compuesta por el déficit primario más el saldo de la deuda en el período anterior más sus respectivos intereses, como se muestra en la siguiente identidad:

$$
B_{t}=\left(G_{t}-T_{t}\right)+\left(1+i_{t}\right) B_{t-1} .
$$

La expresión anterior se pude reescribir como una ecuación de sostenibilidad de la deuda pública, la cual establece que la única forma de reducir la deuda es obteniendo un superávit primario durante el ejercicio.

$$
B_{t}=\left(1+i_{t}\right) B_{t-1}-\left(T_{t}-G_{t}\right)=\left(1+i_{t}\right) B_{t-1}-S P_{t}
$$

Los períodos considerados en estas ecuaciones son, en general, anuales, pero es posible reescalar estas en períodos más cortos dependiendo de la disponibilidad de los datos.

\subsection{Modelo estocástico propuesto}

Con el propósito de medir los efectos de la tasa de depreciación del tipo de cambio sobre el déficit fiscal del GNCC, se modelará dicha tasa como un proceso estocástico de reversión a la media con saltos. En una primera etapa se utiliza un modelo lineal, AR(1), para calibrar la componente de reversión a la media. Es importante destacar que si se utiliza únicamente este tipo de modelos no es posible, en general, describir adecuadamente la dinámica del tipo de cambio, como señalan Jalil y Misas (2007) y Kuan y Liu (1995). En una segunda etapa se agrega un componente de saltos de Poisson para ajustar las innovaciones. Evidentemente, la mezcla de este tipo de especificaciones lineales con saltos se aleja del mundo normal. El modelo propuesto se construye a partir de la ecuación de cálculo del déficit por encima de la línea, es decir, un balance de ingreso y gastos. Sin embargo, a esta ecuación tradicional se le debe realizar

1 En el cálculo por debajo de la línea se tienen en cuenta los ingresos por privatizaciones. 
una desagregación en el pago de intereses, distinguiendo los pagos por endeudamiento interno o externo, con el fin de evaluar los choques de la tasa de depreciación del tipo de cambio. El modelo para el caso de Colombia se desarrollará a partir de la siguiente ecuación, que define el déficit fiscal con la desagregación sugerida:

$$
D_{t}=\left(G_{t}-T_{t}\right)+i_{t} B_{t-1}+i_{t}^{*} B_{t-1}^{*} h_{t}
$$

donde $D_{t}$ denota el déficit nominal para un período, $G_{t}$ corresponde a los gastos sin intereses del gobierno central, $T_{t}$ son los ingresos totales del gobierno central, $i_{t}$ e $i_{t}^{*}$ son las tasas de interés de la deuda pública interna y externa, respectivamente, $B_{t-1}$ y $B_{t-1}{ }^{*}$ son los saldos de la deuda pública interna y externa en el período anterior, respectivamente, y $h_{t}$ es el tipo de cambio nominal. Se define la tasa de depreciación del tipo de cambio como:

$$
e_{t}=\operatorname{In}\left(\frac{h_{t}}{h_{t-1}}\right)
$$

y el cambio porcentual del índice nacional de precios al consumidor o tasa de inflación como:

$$
\pi_{t}=\frac{P_{t}}{P_{t-1}}-1
$$

A partir de las ecuaciones (5)-(7), se llega a una ecuación del déficit fiscal del gobierno central en términos reales:

$$
D_{t}^{\text {real }}=\frac{\left(G_{t}-T_{t}\right)}{P_{t}}+\frac{i_{t} B_{t-1}}{P_{t}}+\frac{i_{t}^{*} B_{t-1}^{*} h_{t}}{P_{t}}-\frac{\pi_{t} B_{t-1}}{P_{t}}+B_{t-1}^{*} h_{t-1}\left(\frac{e_{t}-\pi_{t}}{P_{t-1}\left(1+\pi_{t}\right)}\right)
$$

Después de derivar parcialmente de las ecuaciones (1) y (2) con respecto del tipo de cambio, se tiene que:

$$
\begin{gathered}
\frac{\partial D_{t}}{\partial h_{t}}=i_{t}^{*} B_{t-1}^{*}>0 \\
\frac{\partial D_{t}^{\text {real }}}{\partial h_{t}}=\frac{B_{t-1}^{*}}{P_{t}}\left(i_{t}^{*}+1\right)>0
\end{gathered}
$$


Es decir, un incremento en el tipo de cambio, manteniendo todo lo demás constante, conducirá a aumentos en el déficit fiscal. Para describir el comportamiento de la tasa de depreciación del tipo de cambio, se considera un proceso estocástico de reversión a la media con saltos de Poisson que satisface la siguiente ecuación diferencial estocástica:

$$
d e_{t}=\alpha\left(u-e_{t}\right) d t+\sigma d W_{t}+\eta d N_{t}, d W_{t} \sim N(0, d t), d N_{t} \sim P(\lambda d t)
$$

donde el tipo de cambio, $e_{\mathrm{t}}$ oscila alrededor de su valor promedio de largo plazo $u$, la velocidad de ajuste cuando $e_{t}$ es mayor o menor al promedio $u$ es denotada por $\alpha, W_{t}$ se refiere a un movimiento browniano estándar, $N_{t}$ es un proceso de Poisson homogéneo, $\lambda$ representa la intensidad (número de saltos por unidad de tiempo) del proceso Poisson y $\eta$ corresponde al tamaño medio esperado del salto.

Una vez establecido el proceso estocástico que modela la dinámica estocástica de la tasa de depreciación, se incluye este en las ecuaciones (5) y (8) del déficit fiscal, lo que permite establecer ecuaciones estocásticas del déficit fiscal en términos nominales y reales. No obstante, antes de esto, es necesario suponer el principio de paridad de tasas de interés interna y externa:

$$
i_{t}=i_{t}^{*}+\frac{h_{t}-h_{t-1}}{h_{t-1}}
$$

Es decir:

$$
i_{t}^{*}=i_{t}-e_{t}
$$

Por lo tanto, el déficit en términos nominales satisface:

$$
D_{t}=\left(G_{t}-T_{t}\right)+i_{t} B_{t-1}+\left(i_{t}-\left[\alpha\left(u-e_{t}\right) d t+\sigma d Z_{t}+\eta d N_{t}\right]\right) B_{t-1}^{*} h_{t}
$$

y, en términos reales:

$$
\begin{aligned}
& D_{t}^{\text {real }}=\frac{\left(G_{t}-T_{t}\right)}{P_{t}}+\frac{i_{t} B_{t-1}}{P_{t}}+\frac{B_{t-1}^{*} h_{t}\left(i_{t}-\left[\alpha\left(u-e_{t}\right) d t+\sigma d Z_{t}+\eta d N_{t}\right]\right)}{P_{t}}-\frac{\pi_{t} B_{t-1}}{P_{t}}+ \\
& B_{t-1}^{*} h_{t}\left(\frac{\left[\alpha\left(u-e_{t}\right) d t+\sigma d Z_{t}+\eta d N_{t}\right]-\pi_{t}}{P_{t-1}\left(1+\pi_{t}\right)}\right)
\end{aligned}
$$




\subsection{Estimación de los parámetros del modelo estocástico}

La estimación de parámetros se realizará, por conveniencia, separando los procesos de reversión a la media y de saltos. Estos parámetros serán utilizados en la simulación de Montecarlo. La estimación de los parámetros del proceso de difusión se efectuará a través del método de máxima verosimilitud, como en Moreno (2011). Para ello se parte de la ecuación diferencial estocástica que describe el proceso de reversión a la media utilizado:

$$
d e_{t}=\alpha\left(u-e_{t}\right) d_{t}+\sigma d W_{t}
$$

Si se denota $b=\alpha u$, se obtiene la siguiente expresión:

$$
d e_{t}=\left(b-\alpha e_{t}\right) d t+\sigma d W_{t}
$$

En este caso el lema de Itô conduce a:

$e_{t}=\exp \{-\alpha(t-s)\} e_{t}+\frac{b}{\alpha}(1-\exp \{-\alpha(t-s)\})+\sigma \exp \{-\alpha t\} \int_{s}^{t} \exp \{\alpha v\} d W_{v}$

para $0 \leq s<t$. En consecuencia, si $e_{t}$ se condicionada a una filtración $F_{t}$ se tiene una distribución normal con media y varianza dadas, respectivamente, por:

$$
\begin{gathered}
E\left[e_{t} \mid F_{s}\right]=\frac{b}{\alpha}(1-\exp \{-\alpha(t-s)\})+e_{t} \exp \{-a(t-s)\} \\
V\left[e_{t} \mid F_{s}\right]=\frac{\sigma^{2}}{2 \alpha}(1-\exp \{-\alpha(t-s)\})
\end{gathered}
$$

La siguiente ecuación resulta de la discretización de la ecuación que representa el proceso de reversión a la media (12). Para esta representación se considera una partición $0=t_{0}<t_{1}<t_{2}<\cdots<t_{n}$ con tamaño del paso constante dado por $\Delta t=t_{i}-t_{i-1}$, como en Phillips (1972):

$$
e_{t}=\frac{b}{\alpha}(1-\exp \{-\alpha \Delta t\})+\exp \{-\alpha \Delta t\} e_{t-1}+\sigma \sqrt{(1-\exp \{-2 \alpha \Delta t\}) / 2 \alpha Z}
$$

donde $Z \sim N(0,1)$. Esta expresión se puede simplificar todavía más de la siguiente forma: si se denota 
$c=\frac{b}{\alpha}(1-\exp \{-\alpha \Delta t\}) ; d=\exp \{-\alpha \Delta t\} ; \delta=\sqrt{(1-\exp \{-2 \alpha \Delta t\}) / 2 \alpha}$

Entonces

$$
e_{t}=c+d \gamma_{t-1}+\delta \mathrm{Z}
$$

Considerando una nueva variable $\frac{1}{2}\left(\Delta \hat{\lambda} L_{t} e_{t}+\Delta \hat{\lambda} L_{t-1} e_{t-1}\right)$ y aplicando el método de máxima verosimilitud, se tienen los siguientes estimadores, donde $n$ corresponde al número total de observaciones:

$$
\begin{gathered}
\hat{d}=\frac{n \sum_{i=1}^{n} e_{i}-e_{i-1}-\sum_{i=1}^{n} e_{i} \sum_{i=1}^{n} e_{i-1}}{n \sum_{i=1}^{n} e_{i-1}^{2}-\left(\sum_{i=1}^{n} e_{i-1}\right)} \\
\hat{\theta}=\frac{\sum_{i=1}^{n}\left(e_{i} \bar{d} e_{i-1}\right)}{n(1-\bar{d})} \\
\widehat{\delta^{2}}=\frac{1}{n} \sum_{i=1}^{n} e_{i}-\bar{d} e_{i-1}-\bar{\theta}(1-\bar{d})^{2}
\end{gathered}
$$

Las expresiones anteriores permiten estimar los parámetros del proceso de reversión a la media de tal forma que:

$$
\alpha=-\frac{\operatorname{In}(d)}{\Delta t} ; b=-\frac{\theta \operatorname{In}(d)}{\Delta t} ; \sigma=\frac{\delta}{\sqrt{\left(1-d^{2}\right) / 2 \alpha}}
$$

Un enfoque alternativo para analizar el modelo anterior consiste en partir de una versión discreta de la ecuación (12), es decir,

$$
\Delta e_{t}=\left(b-\alpha e_{t}\right) \Delta t+\sigma \Delta W_{t}, b=\alpha u
$$

Si se escribe $\Delta t=(t+1)-t=1$, entonces $\sigma \Delta W_{t}=\sigma \sqrt{ } \Delta t Z=\sigma Z=\varepsilon_{t}$, donde $\varepsilon_{t}$ es una variable aleatoria normal con media cero y varianza $\sigma^{2}$. En consecuencia,

$$
e_{t+1}=b+(1-\alpha) e_{t}+\varepsilon_{t}
$$


Sean $\beta_{0}=b$ y $\beta_{1}=1-\alpha$, entonces $e_{t+1}=\beta_{0}+\beta_{1} e_{t}+\varepsilon_{t}$. De esta manera se obtiene un proceso $\mathrm{AR}(1)$. Con los estimadores de $\beta_{0} \mathrm{y} \beta_{1}$, se pueden estimar $\alpha, b$ $y \sigma$. En una primera etapa se utiliza este modelo para calibrar el componente de reversión a la media. La tabla 2 muestra los resultados de la estimación de un proceso AR(1) para la tasa de depreciación. Se puede apreciar que el término autorregresivo es estadísticamente significativo aun cuando el ajuste es pobre, ya que se observan errores no normales y exceso de curtosis (algo frecuente en este caso de estimaciones) (ver la figura 6). Se observa también que la serie es débilmente estacionaria, puesto que la raíz del término autorregresivo es menor que 1 en valor absoluto. Dadas las características previas de los errores, en una segunda etapa, se incluye un componente de saltos de Poisson para ajustar las innovaciones en la tasa de depreciación. Claramente, la mezcla de estas distribuciones ya no es normal.

Tabla 2. Calibración del componente de reversión a la media AR(1)

\begin{tabular}{lrrrr} 
Variable dependiente: Rend_TC & & \multicolumn{2}{c}{ Número de observaciones: 1163} \\
\hline \multicolumn{1}{c}{ Variable } & Coeficiente & Error estándar & Estadística $t$ & Prob. \\
\hline C & 0,00021392 & 0,00016209 & 1,31981175 & 0,18715828 \\
AR(1) & 0,20583157 & 0,0142806 & 14,4133656 & $1,90 \mathrm{E}-43$ \\
sigmAsQ & $1,90 \mathrm{E}-05$ & $3,57 \mathrm{E}-07$ & 53,306444 & 0 \\
\hline R-cuadrada & 0,042382 & Media variable dependiente & 0,00021273 \\
R-cuadrada ajustada & 0,04073093 & D. E. variable dependiente & 0,00445833 \\
D. E. de regresión & 0,00436659 & Criterio Akaike info & $-8,02705701$ \\
Suma de residuales cuadrados & 0,02211781 & Criterio Schwarz & $-8,01400776$ \\
Log-verosimilitud & 4670,73365 & Criterio Hannan-Quinn & $-8,02213386$ \\
Estadístico F & 25,6694824 & Estadística Durbin-Watson & 1,95085275 \\
Prob(estadístico F) & $1,23 \mathrm{E}-11$ & AR raíces invertidas & 0,21 \\
\hline
\end{tabular}

Fuente: elaboración de los autores con datos del Banco de la República y del Ministerio de Hacienda.

La figura 6 muestra el comportamiento de los errores, los cuales son no normales con un exceso de curtosis y heterocedasticidad. En este caso, la inclusión de un componente de saltos de Poisson se utiliza para ajustar las innovaciones, como en Akgiray y Booth (1988), Vlaar y Palm (1993), Jiang (1998), Rodríguez y Venegas-Martínez (2010) y Núñez y Ortega (2011). 


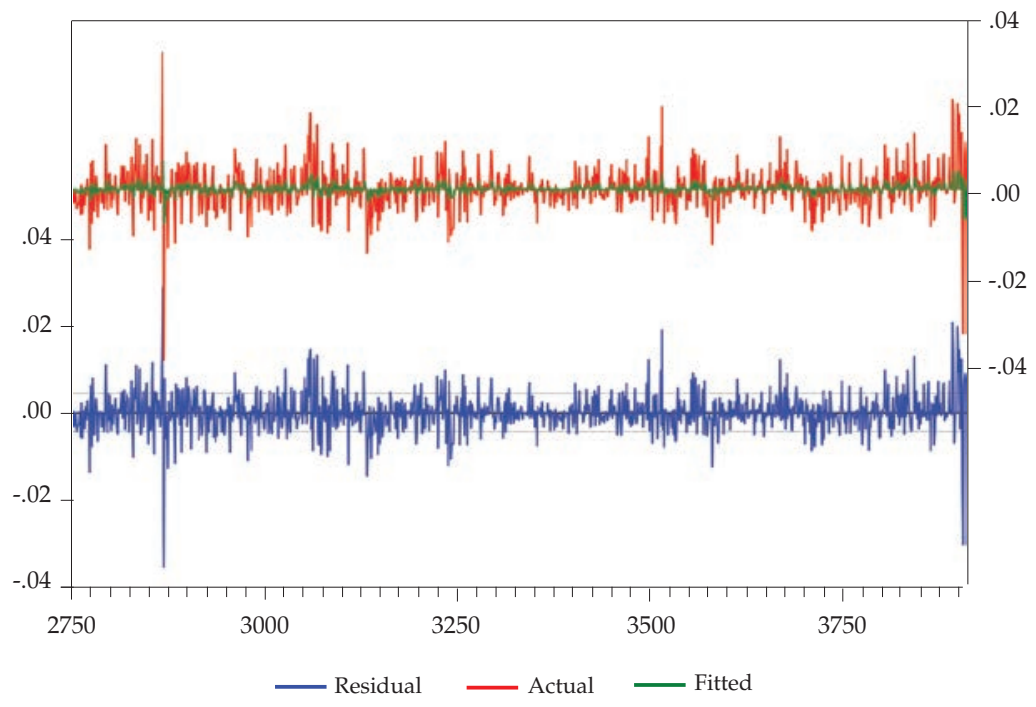

Figura 6. Errores en el componente de reversión a la media AR(1)

Fuente: elaboración de los autores con datos del Banco de la República y del Ministerio de Hacienda.

Tabla 3. Parámetros del proceso de reversión a la media

\begin{tabular}{ll}
\hline$\alpha$ & 0,20583157 \\
$b$ & 0,00021392 \\
$\sigma$ & 0,00436659 \\
\hline
\end{tabular}

Fuente: elaboración de los autores.

Para la estimación de los parámetros del proceso de saltos de Poisson, se sigue la metodología propuesta en el trabajo de Rodríguez y VenegasMartínez (2010), donde se considera que el número de saltos de Poisson por período, $m$, es el número de observaciones que exceden considerablemente tres desviaciones estándar, ya sea por encima o por debajo de la media de un total de $n$ observaciones. La intensidad del salto que se denota por $\lambda$ se calcula como el número promedio de saltos, $m$, por unidad de tiempo. Por último, el tamaño medio esperado del salto $\eta$ se estima como el tamaño promedio de los saltos ajustados por la varianza. De igual forma como se procedió con los parámetros del proceso de reversión a la media, se calculan los valores de los parámetros del proceso de saltos de Poisson, los cuales se resumen en la tabla 4. 
Tabla 4. Parámetros del proceso de saltos de Poisson

\begin{tabular}{cc}
\hline$m$ & 91 \\
$n$ & 3912 \\
$\lambda$ & 0,0232617 \\
$\eta$ & 0,0002556 \\
\hline
\end{tabular}

Fuente: elaboración de los autores.

Con base en los resultados anteriores, se procede a realizar las simulaciones del modelo de la tasa de depreciación del tipo de cambio para posteriormente incorporar los resultados en las ecuaciones estocásticas del déficit fiscal.

\section{Simulación estocástica del déficit fiscal}

Una vez que se tiene el modelo propuesto calibrado, se procede a llevar a cabo las simulaciones por el método de Montecarlo. El primer ejercicio consiste en hacer una simulación de la tasa de depreciación del tipo de cambio para el período de enero de 2000 a diciembre de 2014. En este ejercicio se obtuvieron 1000 trayectorias, las cuales, previamente anualizadas, se incorporaron en la ecuación estocástica del déficit fiscal en términos nominales como proporción del PIB, con el fin de generar trayectorias del déficit. Para la tasa de interés de la deuda interna del gobierno nacional, se utilizó una tasa de interés implícita, la cual es compatible con el planteamiento del modelo propuesto, ya que supone una tasa única para el total de la deuda interna. Esto servirá para implementar el supuesto de paridad de tasas de interés interna y externa. En la figura 7 se observa que la trayectoria promedio simulada del déficit presenta valores altos desde la década final del siglo xx, lo cual es de esperarse, dado que la economía y las finanzas públicas colombianas se encontraban resentidas por la crisis hipotecaria y financiera reciente. Posteriormente, en 2009, se destaca en la simulación el deterioro en el déficit que coincidió con una depreciación del peso colombiano frente al dólar durante los momentos recientes de la crisis financiera estadounidense, cuando se restringió la liquidez en los países emergentes después de que el tipo de cambio nominal estuvo en mínimos durante 2008. En los años siguientes se observa que la tendencia del déficit es a reducirse, lo cual coincidió con un período de apreciación del peso colombiano, motivada por el plan de relajamiento monetario establecido por la autoridad monetaria norteamericana. Así mismo, en la figura 7 la trayectoria promedio simulada se compara con la observada en el período 2000-2015. Es importante destacar que el modelo simulado se aleja 
un poco de las observaciones en los años de la crisis subprime acontecida en Estados Unidos, cuando las variaciones de la tasa de cambio tuvieron valores sustancialmente altos, para el resto del período los resultados cercanos. Esta pequeña desviación en la crisis subprime puede deberse a que el proceso de saltos es un proceso homogéneo. ${ }^{2}$

A continuación, se procede a efectuar un ejercicio de proyección del déficit fiscal del gobierno nacional, lo que permitirá generar una senda proyectada de la deuda pública. Esto será de gran utilidad para el planteamiento de las metas fiscales que se proyectan de manera anual.

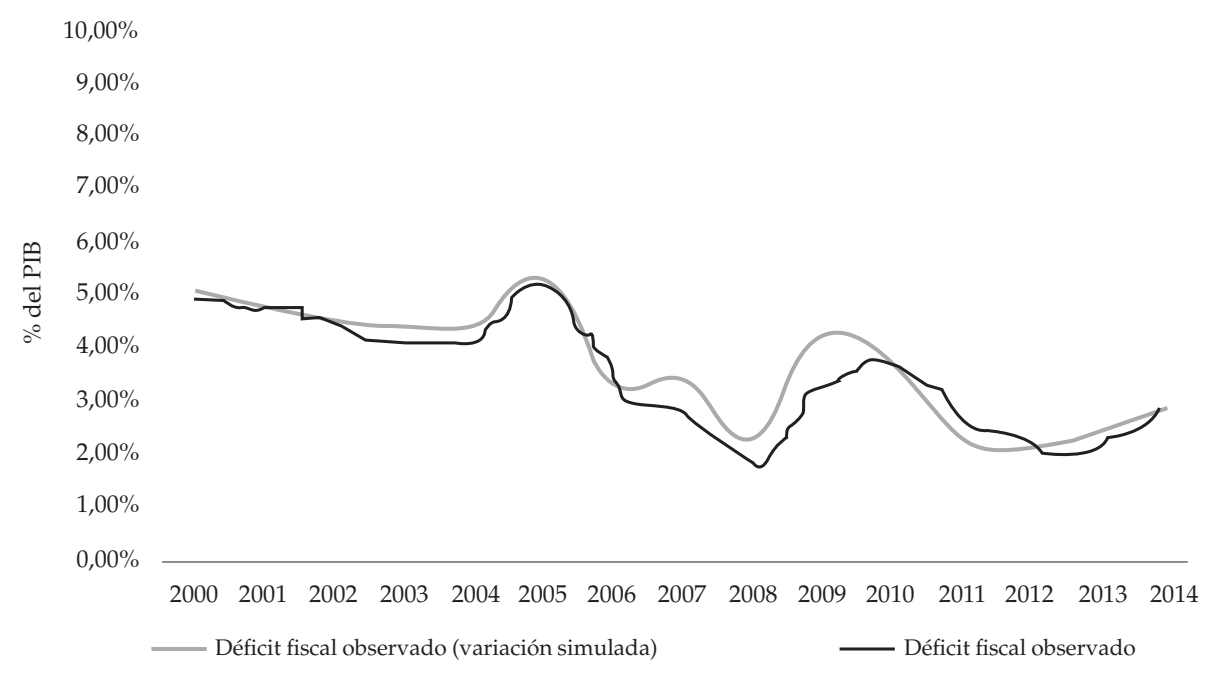

Figura 7. Evolución simulada del déficit fiscal 2000-2014

Fuente: elaboración de los autores con datos del Banco de la República y del Ministerio de Hacienda.

\section{Proyecciones del déficit fiscal y la deuda}

Una herramienta utilizada de manera recurrente para la programación de la política fiscal son las proyecciones de los resultados fiscales. Aunque existen

2 La intensidad del proceso de Poisson se estimó a través del número promedio de saltos por unidad de tiempo. Una intensidad variable que sea mayor en la crisis subprime podría enmendar esta pequeña desviación. Bajo esta alternativa, el modelado y los cálculos de estimación de los parámetros se complican, por lo que esta posibilidad se pospone para investigaciones futuras. No obstante, es importante mencionar que la simulación tiene un buen desempeño a partir de 2010 y esto es esencial para la proyección adecuada de una senda de la deuda del gobierno y la planeación del gasto del GNCC, así como para el establecimiento de metas fiscales. 
diversos modelos que ayudan en esta tarea, la aproximación que se propone en este trabajo mejora el entendimiento sobre la incidencia que tienen las variaciones aleatorias del tipo de cambio en la política fiscal. Para esto se realizan simulaciones de la tasa de depreciación del tipo de cambio peso colombiano-dólar americano. La simulación se lleva a cabo con base diaria y se generan 1000 trayectorias. En este caso, la simulación utiliza 780 días, que constituyen los días hábiles de los años 2015 a 2017. En la figura 8 se ilustran las trayectorias simuladas para la tasa de depreciación del tipo de cambio. El promedio de las trayectorias será el insumo para realizar el cálculo de la ecuación estocástica del déficit fiscal. Como información necesaria para las proyecciones se utilizaron los ingresos y gastos sin intereses estimados para dichos años en el documento de marco fiscal de mediano plazo del Ministerio de Hacienda y Crédito Público colombiano, el cual es la fuente oficial para estas cifras. Como tasa de interés implícita se utilizan los promedios móviles de orden tres. Para el pIB nominal se utilizan las proyecciones del Banco de la República (banco central colombiano).

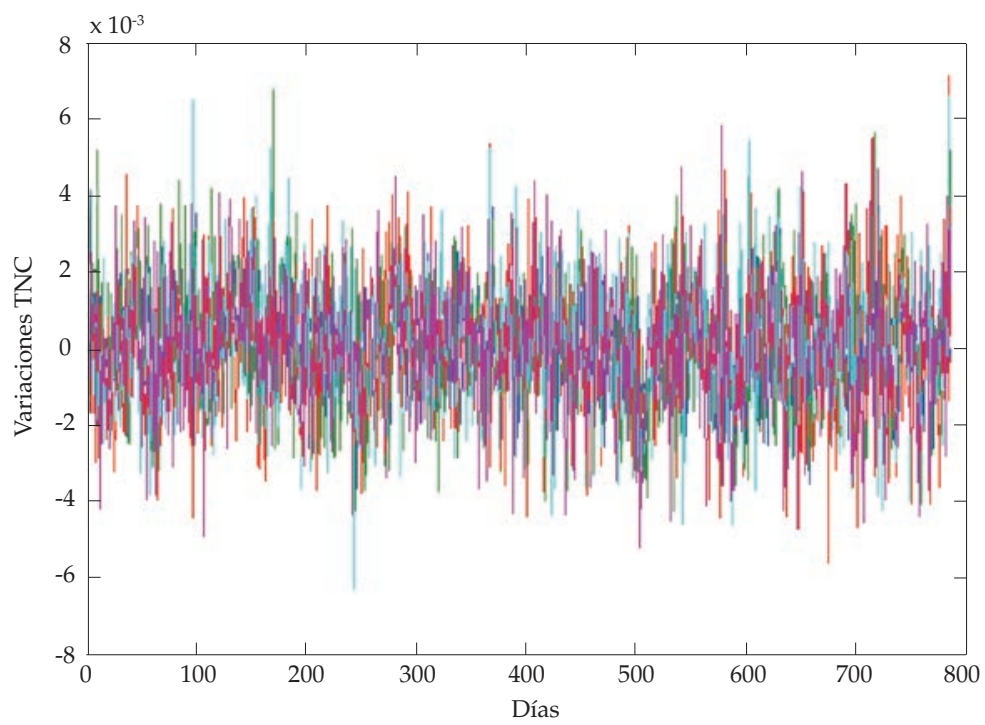

Figura 8. Simulación de la tasa de depreciación del tipo de cambio. Proyección diaria de las variaciones del tipo de cambio de 2015 a 2017

(1000 trayectorias)

Fuente: elaboración de los autores con datos del Banco de la República y del Ministerio de Hacienda y Crédito Público colombianos.

En la figura 9 se presentan los resultados del ejercicio de proyección del déficit fiscal del gobierno nacional entre 2015 y 2017, los cuales fueron cons- 
truidos a partir de la ecuación nominal propuesta en este trabajo. En esta figura se observa que en la tendencia para los próximos años se prevé un deterioro de la situación fiscal del gobierno nacional, lo cual deja entrever una relación con las variaciones positivas simuladas del tipo de cambio. Esto conduce a pensar una vez más que una depreciación del peso está correlacionada con el deterioro en las cuentas fiscales.

Para los años proyectados se estima que el déficit fiscal se encuentre, con un nivel de confianza del $95 \%$, en el rango del 3,2 \% al 3,5\% del PIB para 2015 y del 3,7\% al 3,9\% del PІв para 2016 у 2017. En términos monetarios, el déficit alcanzaría 35 billones de pesos colombianos a finales de 2017. Las proyecciones para 2016 resultaron muy cercanas a los datos observados. Es necesario resaltar que en estas proyecciones se supone como factor de riesgo únicamente la variación del tipo de cambio, ya que el objetivo es simular solo esta variable y estudiar sus efectos en el déficit fiscal.
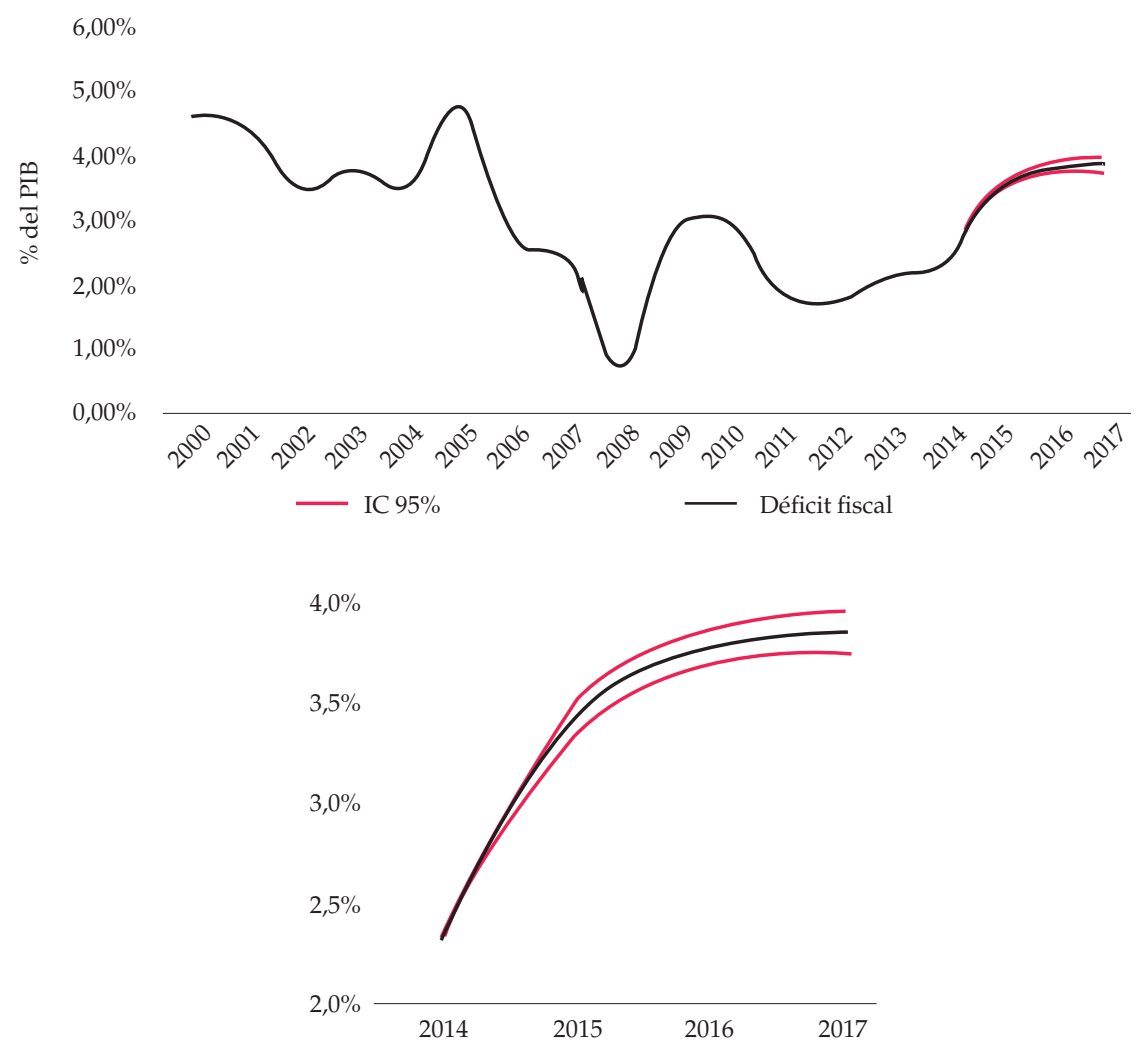

Figura 9. Proyección del déficit fiscal con un nivel de confianza del $95 \%$

Fuente: elaboración de los autores con datos del Banco de la República y del Ministerio de Hacienda y Crédito Público colombianos. 
Por último, en la figura 10 se expone una senda proyectada de la deuda del gobierno nacional, la cual se obtiene haciendo uso de los resultados del ejercicio de proyección del déficit. Este cálculo está basado en la identidad que establece que el déficit por debajo de la línea es equivalente a la variación de la deuda. Una vez que se obtiene el resultado proyectado del déficit, se genera la senda de la deuda, donde se muestra que, con un crecimiento del déficit del gobierno nacional, la deuda va a incrementarse hasta a llegar al $42 \%$ del PIB a finales de 2017.

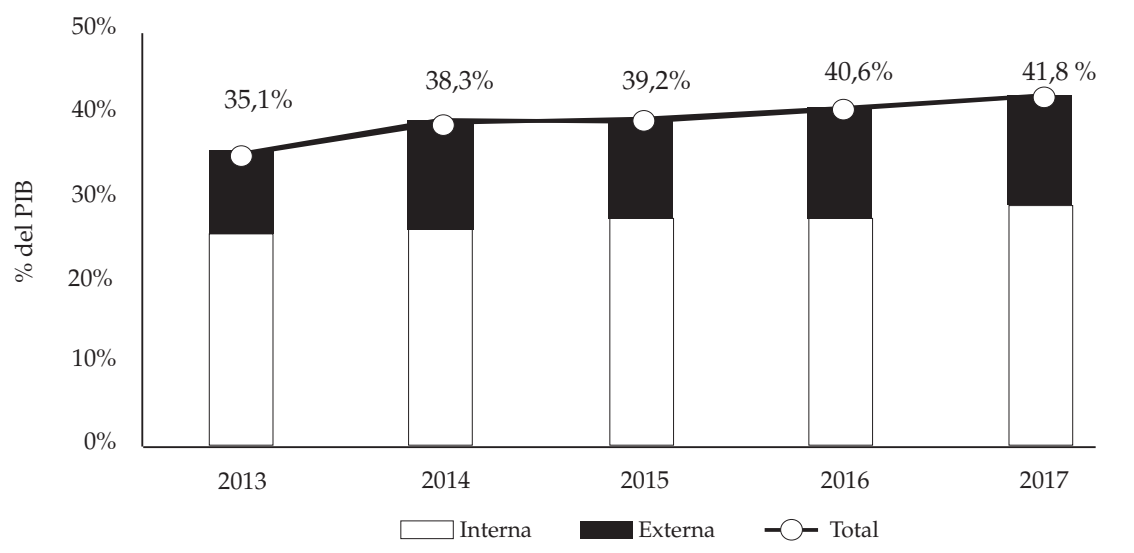

Figura 10. Proyección de la deuda bruta del gncc

Fuente: elaboración de los autores.

\section{Conclusiones}

En esta investigación se presentó un modelo estocástico alternativo para la estimación del déficit fiscal del gobierno nacional colombiano. Las variaciones del tipo de cambio peso colombiano-dólar americano se modelaron como un proceso estocástico de reversión a la media con saltos de Poisson, lo cual abandona el mundo normal (la mezcla normal-Poisson no sigue una distribución normal, sino la de un proceso de Lévy). La tasa de depreciación simulada se incorporó en el cálculo del déficit fiscal. Para la calibración se dividió el proceso en su componente estocástico de reversión a la media y en su componente de saltos de Poisson. El primero se calibró por máxima verosimilitud y el segundo, siguiendo la metodología de Rodríguez y Venegas-Martínez (2010); se simularon 1000 trayectorias entre enero de 2000 y diciembre de 2014. 
Para complementar el ejercicio empírico, se realizaron proyecciones para tres años hacia adelante (2015 a 2017) de las variaciones de la tasa de cambio, con el propósito de generar valores estimados para el déficit fiscal, y se encontró que el déficit para estos años tendría una tendencia a aumentar sus valores dentro del rango del 3,2\% al 3,5\% del pis para 2015 y del 3,7 \% al 3,9\% del PIB para 2016 y 2017, con un nivel de confianza del $95 \%$. Las proyecciones obtenidas para 2016 son muy cercanas a los datos observados. Estos resultados son consistentes con la proyección de variaciones positivas de la tasa de cambio para esos años. Además, como resultados de estas proyecciones se construyó una senda proyectada de la deuda del gobierno nacional, la cual estima que esta llegará a ser el 42\% del PIB a finales de 2017.

Por último, se plantea como una primera extensión de trabajo futuro un modelo que incorpore una estructura de plazos de la tasa de interés (un segundo factor de riesgo), ya que en el modelo empleado se tomó una tasa de interés implícita constante. Este planteamiento incorporaría al modelo la volatilidad de la deuda pública. Otra posible línea de extensión es la consideración de un proceso no homogéneo para describir los saltos que presenta la tasa de depreciación del tipo de cambio. Esto puede enmendar que el modelo simulado no se aleje de las observaciones en los años de la crisis subprime acontecida en Estados Unidos cuando las variaciones de la tasa de cambio tuvieron valores sustancialmente altos.

\section{Referencias}

Aase, K. K. (1988). Contingent claims valuation when the security price is a combination of an Itô process and random point process. Stochastic Processes and Their Applications, 28(2), 185-220.

Agnello, L., Furceri, D., \& Sousa, R. M. (2013). How best to measure discretionary fiscal policy? Assessing its impact on private spending. Economic Modelling, 34(C), 15-24. Dor: http:/ / doi.org/10.1016/j.econmod.2012.10.020

Akgiray, V., \& Booth, G. G. (1988). Mixed diffusion-jump process modeling of exchange rate movements. Review of Economics and Statistics, 70(4), 631-637. Dor: http://doi.org/10.2307/1935826

Ball, C., \& Torous, W. (1983). A simplified jump process for common stock returns. Journal of Financial and Quantitative Analysis, 18(1), 53-65. Dor: http:/ / doi.org/10.2307/2330804

Beinea, M., \& Laurent, S. (2003). Central bank interventions and jumps in double long memory models of daily exchange rates. Journal of Empirical Finance, 10(5), 641-660. 
Bates, D. S. (1988). Pricing options on jump-diffusion process. Rodney L. White Center for Financial Research-The Wharton School University of Pennsylvania. Recuperado de https: / / www.biz.uiowa.edu/faculty / dbates/papers/chapter3.pdf

Bouakez, H., Chihi, F., \& Normandin, M. (2014). Measuring the effects of fiscal policy. Journal of Economic Dynamics and Control, 47(C), 123-151. Dor: http:/ / doi.org/10.1016/j.jedc.2014.08.004

Bouakez, H., \& Eyquem, A. (2015). Government spending, monetary policy, and the real exchange rate. Journal of International Money and Finance, 56(C), 178-201. Dor: http:/ / doi.org/10.1016/j.jimonfin.2014.09.010

Branger, N., \& Larsen, L. S. (2013). Robust portfolio choice with uncertainty about jump and diffusion risk. Journal of Banking and Finance, 37(12), 5036-5047. DoI: http:/ / doi.org/10.1016/j.jbankfin.2013.08.023

Duffie, D. (2001). Dynamic asset pricing theory ( $3^{\mathrm{a}}$ ed.). Princeton University Press. Duffie, D. (2005). Credit risk modeling with affine processes. Journal of Banking and Finance, 29(11), 2751-2802. DoI: http://doi.org/10.1016/j. jbankfin.2005.02.006

Jalil, M. A., \& Misas, M. (2007). Evaluación de pronósticos del tipo de cambio utilizando redes neuronales y funciones de pérdida asimétricas. Revista Colombiana de Estadística, 30(1), 143-161.

Jiang, G. J. (1998). Jump-diffusion model of exchange rate dynamics. Estimation via indirect inference. Working paper. The Netherlands: University of Groningen.

Kuan, C. M., \& Liu, T. (1995). Forecasting exchange rates using feedforward and recurrent neural networks. Journal of Applied Econometrics, 10(4), 347-364.

Merton, R. C. (1976). Option pricing when underlying stock returns are discontinuous. Journal of Financial Economics, 3(1-2), 125-144.

Moreno, J. (2011). Estimación de parámetros en ecuaciones diferenciales estocásticas aplicadas a finanzas. Odeon, 6, 131-144.

Nag, A. K., \& Mitra, D. A. (2002). Forecasting daily foreign exchange rates using genetically optimized neural networks. Journal of Forecasting, 21(7), 501-511. DoI: https: / / doi.org/10.1002/for.838

Ni, H., \& Yin, H. (2009). Exchange rate prediction using hybrid neural networks and trading indicators. Neurocomputing, 72(13-15), 2815-2823. DOI: https: / / doi.org/10.1016/j.neucom.2008.09.023

Núñez, J. A., \& Ortega, E. (2011). Continuous time models of interest rate: testing peso-dollar exchange rate. Economía: Teoría y Práctica, (34), 43-63.

Penati, A. (1983). Expansionary fiscal policy and the exchange rate: a review. Staff Papers. International Monetary Fund, 30(3), 542-569. 
Phillips, P. C. B. (1972). The structural estimation of a stochastic differential equation system. Econometrica, 40(6), 1021-1041.

Rodríguez, A., \& Venegas-Martínez, F. (2010). Efectos del tipo de cambio sobre el déficit público: modelos de simulación de Montecarlo. Revista Contaduría y Administración, 232, 11-40.

Tenti, P. (1996). Forecasting foreign exchange rates using recurrent neural networks. Applied Artificial Intelligence, 10(6), 567-581.

Vallejo-Jiménez, B., \& Venegas-Martínez, F. (2017). Optimal consumption and portfolio rules when the asset price is driven by a time-inhomogeneous Markov modulated fractional brownian motion with multiple Poisson jumps. Economics Bulletin, 37(1), 314-326.

Venegas-Martínez, F. (2001). Temporary stabilization: a stochastic analysis. Journal of Economic Dynamics and Control, 25(9), 1429-1449. Dor: http:/ / dx.doi.org/10.1016/S0165-1889(00)00044-0

Venegas-Martínez, F. (2006). Stochastic temporary stabilization: undiversifiable devaluation and income risks. Economic Modelling, 23(1), 157-173. DoI: http://dx.doi.org/10.1016/j.econmod.2005.09.004

Venegas-Martínez, F. (2009). Temporary stabilization in developing countries and real options on consumption. International Journal of Economic Research, 6(2), 237-257.

Venegas-Martínez, F., Cruz-Aké, S., \& López-Herrera, F. (2013). Pricing contingent claims with an underlying asset driven by an extreme value distribution: options on Dow Jones Industrial Average index, 2009-2010. Frontiers in Finance and Economics, 10(2), 63-84. 